\title{
On the Fundamental Tautology of Validating Data-Driven Models and Simulations
}

\author{
John Michopoulos and Sam Lambrakos \\ Materials Science and Technology Division, \\ U.S. Naval Research Laboratory, Washington, DC. 20375, U.S.A \\ \{john.michopoulos, lambrakos\}@nrl.navy.mil
}

\begin{abstract}
Recent advances in Dynamic Data Driven Application Systems (DDDAS) facilitated by the present level of computational technologies, as well as advances in data-driven modeling and simulation, impose the need for a critical evaluation of paradigms underlying Qualification, Validation and Verification (QV\&V). This paper discusses the fundamental irrelevance of conventional validation procedures with respect to data-driven models and simulations. This inherent property of data-driven models and simulations makes the data-driven approaches extremely desirable from a reliability perspective. An informal comparison of the logical flow of traditional and evolved QV\&V demonstrates the tautological nature of data-driven model validation. A brief epistemological review of the origins of traditional and evolved $\mathrm{QV} \& \mathrm{~V}$ is also presented.
\end{abstract}

\section{Introduction}

The need for high fidelity simulation of realistic size and complexity systems have motivated studies concerning methodologies for the development of Dynamic Data Driven Application Systems (DDDAS) by various groups. The National Science Foundation has taken a leadership role in fostering research on such efforts through the initiative on DDDAS [1].

In the past, aspects of DDDAS and dynamic data in particular, have been used to reduce the effect of uncertainties on weather prediction modeling methods, and correct error propagation to improve the fidelity of model predictions. In addition, data-driven aspects of DDDAS have also been used in the past for very successful material characterization.

Recent advances in DDDAS facilitated by the present level of computational achievements, as well as advances in data-driven modeling and simulation, impose the need for a critical evaluation of paradigms underlying Qualification, Validation and Verification $(\mathrm{QV} \& \mathrm{~V})$. This paper focuses on the fundamental irrelevance of conventional validation procedures with respect to data-driven models and simulations. This follows since conventional validation procedures are based on paradigms and associated terminology, that are historically biased and related to concepts and implementations that do not correspond to today's advanced computational practices and possibilities. 
Additional areas of contemporary interest where there is a need for dynamic data-driven Modeling and Simulation (M\&S) of systems are multi-physics systemic behavior prediction, multi-scale fire dynamics prediction, disaster management decision support, bio-chemical contaminant dispersion prediction, hydrogen storage, welding and heat deposition processes, artificial muscle autonomous systems, fuel cells, soft robotics and nano-robotics applications, to name only a few.

There are formal aspects of "data-driven system modeling" that are related to methods of "system identification" and "parameter estimation," which are based on formal systems theory. In the discussions that follow, the concept of system identification has been extended to include that of data-driven modeling. Further, "dynamic" data driven is to be understood as implying system identification within the context real-time input of data, which is considered as having been obtained most recently from sensors. Similarly, the use of most recently obtained data for adaptive simulation control or steering, constitutes the second use of the term "dynamic data driven" as has been discussed elsewhere $[2,3,4]$.

A key aspect of our development concerns the concept of "weak termination" that implies informally the incomplete character of validation with respect to the predictive capability of a model. A rigorous examination of the mathematical foundations of this concept is beyond the scope of this presentation in that it relates to temporal and ontological formalisms of logical meta-systems [5].

In this paper, we first present the definitions and origins of the particular QV\&V terms. We subsequently identify how weak termination undermines the fidelity, accuracy and reusability of models. We further demonstrate how the application of data-driven M\&S avoids weak termination and enables reusability of models and simulations. We conclude with a presentation of the association of scientific methods with forward and inverse (data-driven) modeling, indicating that forward modeling is an artifact of practices based on applying the hypotheticodeductive method. These practices are not necessary when behavioral data can be collected and utilized for M\&S.

\section{Qualification, Validation and Verification}

Model fidelity, accuracy, and high confidence in predictability requires that contemporary M\&S are subject to various QV\&V procedures. There are many descriptions of how QV\&V relates to modeling and simulation. Figure 1 represents the modeling and simulation process along with the associated QV\&V in terms of logical flow. This logical flow represents a unification of the abstractions defined by many organizations such as the AIAA [6], ASME [7], DoD's Defense Modeling and Simulation Office (DMSO) [8] and DOE Defense Program's (DOE/DP) Accelerated Strategic Computing Initiative (ASCI) [9]. The dotted arrows shown in this figure represent human activities that are implemented with various degrees of automation, allowing a transitioning from the physical system to the system's conceptual model (via analysis), next to the computational model (via computational methods), and then back to the physical system (via simulation). 
A conceptual model is constructed by analysis of the behavioral structure of the physical system within an application context. The conceptual model can be a set of partial differential equations (PDEs) representing conservation laws with appropriate constitutive equations. Typically, this type of mathematical representation is known as an analytical model that encapsulates the conceptual model. This model reproduces the behavior of the physical system and belongs to the class of models designated as "explicit" or "physics-aware" [10].

Another form of conceptual model is that of a "physics-agnostic" model such as rule-based (e.g. Cellular Automata or Genetic Algorithms) or input-output associator technologies (e.g. Neural Nets, Perceptrons, Support Vector Machines). The corresponding analytical or mathematical model introduces errors associated with its underlying idealized assumptions.

The computational model is encapsulated by the software that implements the conceptual model within the computational infrastructure. It is constructed by a variety of programming techniques based on various degrees of computational automation ranging from manual to automated software generation that exploits the ability of commercial design tools. The computational model justifies the need for verification because it introduces additional uncertainty that is associated with space and time discretization, i.e. errors associated with the discrete representation of differential operators and machine truncation.

The formal definitions of the QV\&V terms are as follows [6]:

- Qualification is the process of determining that a conceptual model implementation represents correctly a real physical system.

- Verification is the process of determining that a computational model implementation represents correctly a conceptual model of the physical system.

- Validation is the process of determining the degree to which a computer model is an accurate representation of the physical system from the perspective of the intended uses of the model.

Both validation and qualification attempt to establish the representational fidelity of the conceptual (qualification) and computational (validation) models relative to the physical system. For this reason, qualification may be considered another form of validation. This consideration explains why most of the bibliography concerns verification and validation $(\mathrm{V} \& \mathrm{~V})$. Verification attempts to determine the error and accuracy between two models (conceptual and computational). The modeling of uncertainty has to address the error originating from various sources extending from sensor device noise to algorithmic approximation and computational implementation of mathematical primitives by particular hardware architectures.

Shown in Fig. 1 are QV\&V concepts in terms of a comparison between the behavior of the physical system (via experimentation), and the conceptual and computational systems (via simulation). In addition to complexity, the most critical issue associated with this data-driven behavioral comparison among the various system representations is "weak termination". Unfortunately, termination of the recursive process of minimizing the differences between model predicted data sets and data sets generated by the physical system, is not guaranteed for 


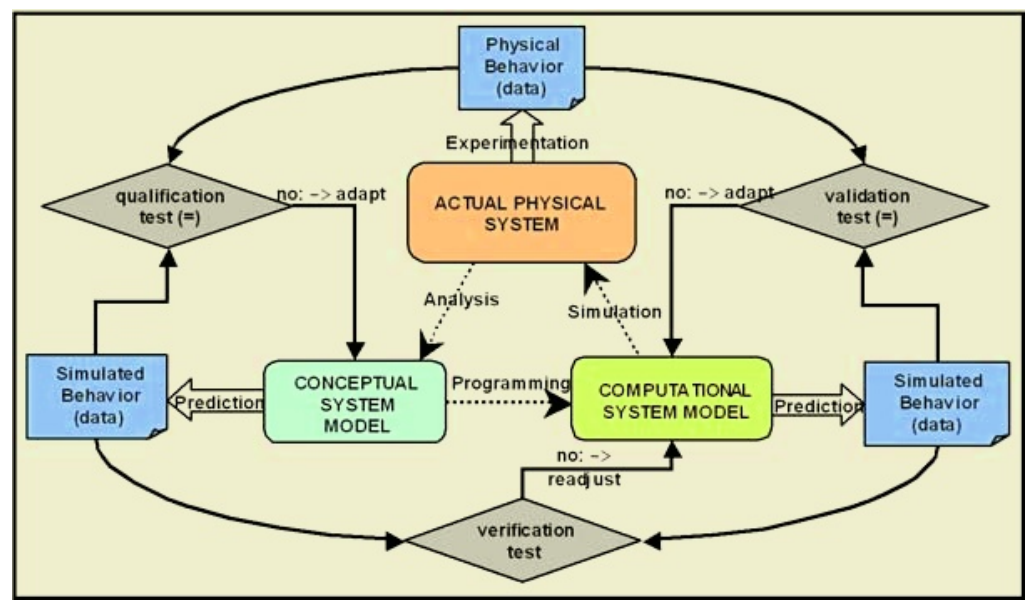

Fig. 1. Traditional QV\&V for the M\&S process

the user defined accuracy (as a measure of fidelity of simulation) in a finite time, nor is it guaranteed that there is a unique model prediction that converges to the physical system behavior with a desirable speed. This is represented clearly by the fact that as long as the comparison modules in Fig. 1 evaluate to "no," the methodology will continue to iterate. This situation forces the user in general, to employ "engineering approximations." That is to say, attitudes of accepting models within "acceptable bounds" for specific applications, which consequently lead to a plethora of low-confidence models that vary according to the personal modeling assumptions of the specific user.

\section{Embedding Validation into Modeling and Simulation}

Addressing weak termination as well as moving towards a realistic approach that guarantees relative fidelity in a model requires considering an alternative approach. This approach involves implementing an M\&S methodology that can utilize: (1) data-streams of controlled continuous system behavior (stimulusresponse pairs of all observables regardless of their field or non-field nature); (2) analytical representations of the model that can accurately reproduce a subset of the acquired data via system identification through successive dynamic model adaptation with the help of optimization techniques (encoding intrinsically the validity of the derived model); and (3), derived models for simulating the predictive response of the originally modeled system or any other system that shares the identified continuous behavior with the original system.

Figure 2 shows the proposed data driven M\&S process as a modification of that shown in Fig. 1. The modules located within the dashed-line region constitute an alternative conceptual model that is defined by the optimization structure comprising these modules. This approach effectively embeds data asso- 


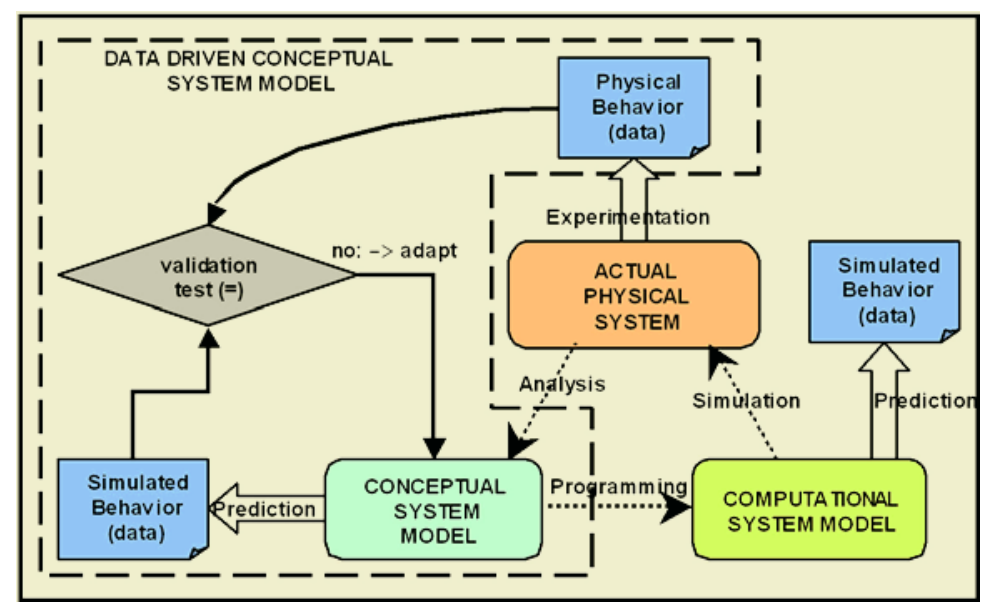

Fig. 2. Evolved QV\&V for data-driven M\&S process

ciated with observed behavior of the physical system, into the conceptual model, thus guaranteeing the intrinsic validity of the conceptual system. For continuous systems, this model usually refers to the mathematical formulation representing the conservation laws and associated constitutive equations. Since no comparative module exists between the physical and simulated data (see Fig. 2) this methodology eliminates entirely the weak termination problem. Termination is strong because the adaptively computed model is trained on the behavioral data and therefore must terminate if it satisfies the optimization criteria, including any acceptable error tolerance between actual and simulated data. Accordingly, termination occurs prior to using the model for prediction of behavior.

\section{Epistemological Origins of QV\&V in M\&S}

A more detailed examination of the logic underlying $\mathrm{V} \& \mathrm{~V}$ in traditional $\mathrm{M} \& \mathrm{~S}$ as shown in Fig. 1, indicates a paradigm for prediction that is based on the hypothetico-deductive scientific method (HDSM) [11], and that is applied by the majority of researchers. This paradigm was first applied by Newton $[12,13]$, who established the foundation for the Kantian reconciliation of the Cartesian rationalism with the Baconian empiricism [11]. Its intermediate stages were marked by the influence of positivism that was based on Compte's strict empiricist claims that valid knowledge is only that which is based on experience $[12,13]$. The "Vienna Circle" of scientists and logicians, further contributed by establishing the logical positivism aspect of the method, which accepted Wittgenstein's [12] verification theory of meaning that considers as meaningful only those prepositions (theories) that can be empirically verified.

The HDFM program involves the steps of making an original observation of a physical system's behavior, making a hypothesis (i.e. making a highly idealized 
abstract model) that may explain (predict) the observation, formulating a theory to be used for predictions, and finally generating observations via experiments for the purpose of verifying [14] or falsifying [15] the theory. If the experiment verifies the theory, then the theory can be considered valid until another experiment renders it false. Further softening of these attitudes resulted from the Logical Empiricism of Carnap [16] that replaced the idea of verification with the idea of "gradually increasing confirmation." That is to say, if the experiment falsifies the theory, then one must change the hypothesis and/or the theory and then reattempt validation. The most distinctive and highly argued [15] characteristics of a theory generated by this approach, are that it is tentative, non-permanent and iterative. These characteristics imply the presence weak termination as identified earlier for the M\&S. There is a strong tendency in the literature to link Popper's falsification idea to the inductive scientific method leading to inverse modeling. From our perspective however, since weak termination is inherently present (i.e. if a contradictory experimental result appears, then the model is false), we have considered this approach as just a more robust HDSM than that of Hempel [10].

As an alternative to a strict adherence to a single scientific method, Critical Relativism advocates [17] that there is no single scientific method. Instead, disciplinary knowledge (as exercised through particular scientific methods) is to be viewed as contingent upon the beliefs, values, standards, methods and cognitive aims of its practitioners. Consequently, critical relativism rejects the aspect of positivism that implies that there is a single knowable reality to be discovered via the scientific method [18]. This relativist view permits the transcendence of the inductivist idea of Roger and Francis Bacon, that was recommended (but not practiced) by Newton and systematized by Herschel $[12,13]$. Accordingly, this idea tunneled trough HDFM practices, and into the middle sixties at the Naval Research Laboratory (NRL) as an industrialized inductive pragmatic scientific method (IIPSM) [10, 19, 20].

At NRL, the advent of coupling hydraulically controlled mechanical power technology with that of computational management and control in the area of testing machines as efficient empirical fact collectors provided the industrialized character of efficiency through automation. The inductive character of the method was provided by insisting that only theories and/or models of continuum system behavior that are consistent with the facts be considered as their functional abstractions. The pragmatism of Pierce and James [12] provided the scope for such a method by enforcing the idea that what matters is not the structural elegance of a theory or model, but instead, the confidence and fidelity of prediction of a theory/model that makes it suitable for application.

The IIPSM program involves the massive automated acquisition of experimental measurements of a physical system's behavioral response. This is followed by construction of a theory in the form of a specific mathematical representation with adjustable parameters that are capable of encoding system behavior. The values of these parameters are assigned by minimizing the error between predicted and measured data sets. Finally, the theory is used for predicting system response given the condition that the stimulus to behavior (or input to the 
system) is within the bounds of the original experimentally observed data-set. it follows that for this approach theory/model validation is inherent (since it has been constructed to reproduce the observed data) and therefore weak termination of its verifiability is not relevant.

In the case of HDSM we start with a small amount of data (or no data at all) that represents casual empirical observations, then we generate a plausible theory/model (a set of equations associated with a given axiomatic foundation), then we generate a predicted-behavior data set (simulation), and finally we perform experimentation to collect the actual behavior data set, in order to compare the two. This implies a logical flow from theory to prediction and then to data. In the sense that we are marching forward from theory to data, this approach has also been referred to by various investigators as the "forward," "theory-driven" or "traditional" approach. On the other hand, the IIPSM implies a logical flow from data to theory, and then to prediction. Relative to the directionality of logical flow between data and theory, this approach is inverse to the HDSM and is termed the "data-driven" approach.

\section{Conclusions}

Our discussion has brought to light significant aspects of validation as they relate to $M \& S$. In particular, that validation is actually not needed by those who pursue data-driven adaptive $M \& S$ methods because it is an inherent property of these methods. This inherent validation makes data-driven methods very desirable from a reliability perspective and suggests that when enough data can be collected then data-driven methods should be considered favorably.

\section{Acknowledgments}

The authors acknowledge the support by the National Science Foundation under grant ITR-0205663 and express their gratitude to Dr. F. Darema for her support of our DDDAS-related activities. Partial support from NRL's 6.1 core-funding is also greatly acknowledged.

\section{References}

1. Darema, F., Dynamic Data Driven Applications Systems: A New Paradigm for Application Simulations and Measurements. International Conference on Computational Science - ICCS'04, Krakow, Poland, June 6-9, 2004, Part III Series: Lecture Notes in Computer Science, Bubak, M.; Albada, G.D.v.; Sloot, P.M.A.; Dongarra, J. (Eds.), LNCS 3038, (2004), 662-669.

2. Michopoulos, J., Tsompanopoulou, P., Houstis, E., Rice, J., Farhat, C., Lesoinne, M., Lechenault, F., DDEMA: A Data Driven Environment for Multiphysics Applications,in: Proceedings of International Conference of Computational Science ICCS'03, Sloot, P.M.A., et al. (Eds.) Melbourne Australia, June 2-4, LNCS 2660, Part IV, Springer-Verlag, Haidelberg, (2003), 309-318. 
3. Michopoulos, J., Tsompanopoulou, P., Houstis, E., Farhat, C., Lesoinne, M., Rice, J., Joshi, A., On a Data Driven Environment for Multiphysics Applications, Future Generation Computer Systems, in-print (2005).

4. Michopoulos, J., Tsompanopoulou, P., Houstis, E., Joshi, A., Agent-based Simulation of Data-Driven Fire Propagation Dynamics, Proceedings, International Conference Computational Science - ICCS'04, Krakow, Poland, June 6-9, 2004, Part III Series: Lecture Notes in Computer Science, Bubak, M.; Albada, G.D.v.; Sloot, P.M.A.; Dongarra, J. (Eds.), LNCS 3038, (2004), 732-739.

5. Van Benthem, J.F.A.K, The Logic of Time, D. Reidel Publishing Company, Doordrecht, (1949).

6. American Institute of Aeronautics and Astronautics Standards Program, Guide for the Verification and Validation of Computational Fluid Dynamics Simulations, AIAA report G-077-1998, (1998).

7. ASME-JFE, Journal of Fluids Engineering Editorial Policy Statement on Control or Numerical Accuracy, Jnl. Of Fluids Engineering, Vol. 115, 3, (1993), 339-340.

8. DoD, Verification, Validation, and Accredization (VV\&A) Recommended Practices Guide, Defense Modeling Simulation Office, Office of the Director of Defense Research and Engr., available: www.dmso.mil/docslib

9. M. Pilch, T. Trucano, J. Moya, G. Froelich, A. Hodges, D. Peercy, Guidelines for Sandia ASCI Verification and Validation Plans - Content and Format: Version 2.0, Sandia Reports SAN2000-3101, (2001).

10. Michopoulos, J.G., Mechatronically Automated Characterization of Material Constitutive Response, in Proceedings of the 6th World Congress on Computational Mechanics (WCCM-VI), September 5-10 2004, Beijing China, Tsinghua University Press and Springer, (2004) 486-491.

11. P. Thagard, Computational Philosophy of Science, MIT Press, Cam- bridge, MA, (1988).

12. Cooper, D.E., Epistemology: The Classic Readings, Blackwell Publ., Oxford, (1999).

13. Losee, J., A Historical Introduction to the Philosophy of Science, Oxford Un. Press, Oxford (2001).

14. C. G. Hempel, Aspects of Scientific Explanation, Free Press, New York, (1965).

15. K. Popper, Unended Quest, Fontana, London, 1976.

16. Carnap, R., The Philosophy of Science, New York, NY: Basic Books, (1966).

17. Anderson, P.F., Marketing, Scientific Progress, and Scientific Method, Journal of Marketing, 47, (Fall), (1983), 18-31.

18. Olson, J.C., Towards a Science of Consumer Behavior, in Advances in Consumer Research, Vol. 9, ed. Andrew A. Mitchell, Ann Arbor, MI: Association for Consumer Research, v- x, (1981).

19. Michopoulos, J.G., Computational and Mechatronic Automation of Multiphysics Research for Structural and Material Systems, Book Chapter, in "Recent advances in Composite Materials" in honor of A.A. Paipetis, Kluwer Academic publishing (2003).

20. Lambrakos, S.G., and Milewski, J.O., Analysis of Processes Involving Heat Deposition Using Constrained Optimization, Sci. and Tech. Of Welding and Joining, 7 (3), (2002), 137. 